\title{
A Study of Metaphorical Culture of "Head"
}

\author{
Man Zheng \\ School of Foreign Languages, Guizhou Normal University, Guiyang, 550001, China
}

\begin{abstract}
Metaphor exists everywhere, which is not only a figurative use of language, but also a cognitive tool and a way of thinking. "Head" has a lot of metaphorical senses in English, for instance, "To hit a ball with your head" and "The side of a coin that has a picture of someone's head on it". These unique metaphorical senses are closely related to the football and coin culture in foreign countries. Taking the different senses of "Head" as the object of study, this paper analyzes the generation and cognitive styles of the metaphorical senses of "Head" from the perspective of Conceptual Metaphor Theory, and further explores the cultural factors behind these metaphorical senses of "Head".
\end{abstract}

Index Terms — head, conceptual metaphor theory, metaphorical senses, cultural factors

\section{INTRODUCTION}

"Head" is an ordinary lexicon in English vocabulary, which has many metaphorical senses. Cognitive linguists hold that metaphor is not only a linguistic phenomenon, but also an important way of recognizing the world, which exists everywhere in people's daily communication and social life. Conceptual metaphor refers to understanding one conceptual domain in terms of another conceptual domain in essence.

The Multiple senses of "Head" can be regarded as the result of metaphorical cognition, which implies that there is a process in which the cognition of a word extending from the central or basic sense to others. Metaphor acts as a bridge which enables a specific word to generate numerous semantic meanings. Metaphorical cognition is grounded upon the systematic correlations within human experience. On the one hand, people of different nations may have similar experience in many aspects thus similar or identical metaphors may produce in different languages. On the other hand, Metaphors in different languages have their own characteristics due to the differences of time and space, region, natural environment and cultural tradition from one nation to another (Dai \& Gao 2007). Metaphorical cognition in each language shows profound cultural details of that country (Zhang, 2004).

The existed studies of metaphor mainly focus on the general description of linguistic metaphorical phenomena in specific languages, such as Gao Xiaorong (2006), Xiao Junmin (2013), Dai Weiping (2014). There are few in-depth studies on the source of metaphorical cognition and few researches on the metaphorical mechanism and the potential cultural factors involved in a certain metaphor. Taking the metaphorical senses of "head" as the object of research, this paper analyzes the cognitive styles of "head" from the perspective of Conceptual Metaphor Theory, further explores the cultural factors behind the metaphorical cognition of "head". Readers are expected to have a better understanding of the metaphorical senses of "head" and get aware of the distinctive cultural factors which drive western people's cognition of "head". Meanwhile, this paper aims to provide some ideas for the study of western metaphorical cognition of vocabulary.

\section{A BRIEF INTRODUCTION OF CONCEPTUAL METAPHOR THEORY}

The concept of conceptual metaphor was first put forward by Lakoff and Johnson in their book Metaphor we live by. Before that, metaphor has long been taken as a linguistic phenomenon and studied as a rhetorical device. Lakoff regarded metaphor as a way of thinking, he argued that metaphor is pervasive in everyday life, not just in human language, but also in human thought and action, human's ordinary conceptual system, in terms of which man both think and act, is fundamentally metaphorical in nature (Lakoff \& Johnson, 1980, P.4).

Lakoff and Johnson further divided conceptual metaphor into three types: structural metaphor, orientational metaphor and ontological metaphor. Structural metaphor refers to the cases where one concept (The target concept) is metaphorically structured in terms of the other (The source concept). The two concepts belong to different cognitive domains and there is a regular correspondence between the constituents of them. For instance, in the expression: "time is money", "time" and "money" are concepts of two cognitive domains, and there are some coherent relations between them: both "time" and "money" are valuable, available for using or occupation and can be saved or wasted. These properties makes it possible for people to understand the invisible and abstract domain of "time" with the conceptual domain of "money" which is concrete and tangible.

Orientational metaphor refers to understanding abstract concepts like emotions and feelings according to some spatial orientation words (such as up and down, inside and outside, front and back, center and edge, etc.) based on the basic experience of human spatial orientation. In the expressions of orientational metaphor: "I'm in high spirits" and "I'm feeling down", the abstract emotional concepts of "happiness" and "sadness" are expressed in terms of the locative words "high" and "down" respectively, which entails the conceptual metaphor that "happy is up" and "sad is down". 
Ontological metaphor shows that people express and understand intangible concepts such as ideas, emotions, psychological activities, and the state of events based on people's experience with physical objects (especially their own bodies) in the physical world. Ontological metaphor is widely used in human language, for instance, in the expression of "His fear of insects is driving him crazy", the abstract concept of "fear" is described as a concrete and tangible entity which can make the person involved frightened.

Contemporary cognitive linguistics holds that metaphor is not just a matter of language, that is of mere words, human thought is largely metaphorical. The metaphorical expressions we realized in our daily life are only the superficial manifestations of the metaphorical cognitive system and metaphorical expressions are fundamentally dominated by conceptual metaphors (Cheng \& Zeng, 2003).

\section{An ANALysis Of THE SENSES OF "HeAD” IN ENGLiSH}

"Head" is a typical polysemy, which can be a noun, an adjective as well as a verb. "head" possess a core sense, from which the other senses are derived by virtue of metaphorical cognition, and each sense is merely suitable for a particular context (Deng, 2013). The general senses of "head" in Oxford Advanced Learner's English-Chinese Dictionary (2014) are listed as follow:

(1)The part of the body above the neck where the eyes, nose, mouth, ears, and brain are.

(2)A person or animal when considered as a unit.

(3)A measure of length or height equal to the size of a head.

(4)The mind and mental abilities.

(5)Someone in charge of or leading an organization, group, etc.

(6)The side of a coin hat has a picture of someone's head on it.

(7)The top part or beginning of something.

(8)The part of a tape or video recorder that touches the tape to record and play music, speech, etc.

(9)The main part of the phrase, to which the other parts are related.

(10)Geography a narrow area of high land that sticks out into the sea.

(11)To go in a particular direction.

(12)To be in charge of a group or organization.

(13)To hit a ball with your head.

(14)To be at the front or top of something.

(15)To lead or control something.

(16)The layer of small white bubbles on the top of a glass of beer

(17)Main or most important.

As for the senses listed above, sense (1) can be regarded as the core sense of "Head", to which sense (3) is closely related and other senses are all derived from, and Each sense of head has the best suitable context for use (Deng, 2013). Sense (2) and (11) are raised due to the cognitive mechanism of metonymy, the rest can be all attributed to the cognitive mechanism of metaphor. Sense (9) and (10) can be further classified into the senses produced under the cognitive mechanism of structural metaphor, Sense(5), (7), (8), (12), (13), (14), (15) and (16) can be regarded as the productions of orientational metaphor and Sense (4), (6), (17) the results of ontological metaphor.

The metonymic cognition of "head" works based on the fact that head is a vital human part, without it, the whole body will cease to live. Therefore, "head", the salient part of human body, is frequently used to point the whole person through the cognitive mechanism of conceptual metonymy. For instance, in sense (2) and (11), "head" is used to represent the whole human body, on the other word, "head" provides the mental process to the cognition of the whole person and the person who are moving towards a particular direction.

About the cognitive mechanism of Conceptual Metaphor, there are two cognitive domains in sense (10), namely the cognitive domain "head" and that of "cape", and the coherent structural relationship exited between them: the cape is protruding from the sea just like a human "head" above the shoulders in appearance. The same to sense (9), "head" and "key word" belong to different conceptual domains, the similarities between them, that is the importance of the "keyword" in the whole sentence and the significance of the "head" organ to the whole human body provide the basis for people's metaphorical cognition, then "head" is endowed with the meaning of "the key word".

As the top part of human body, the spacial feature of "head" gives rise to a series of metaphorical senses. For instance, the concepts of "the top position of the object" in sense (7), "the top authority" in sense (15) and "the top of a glass of beer" in sense (16) are all derived from the position feature of "head" based on people's orientation experience in physical world (Cheng \& Zeng, 2003).

People's experiences with physical objects(their bodies in particular) serve as a foundation of the realization of ontological metaphors. In sense (4) and (17), abstract concepts of "mental abilities" and "significance" are both viewed as the equivalent of "head", that is, an entity and a concrete substance.

\section{The Cultural Factors of Metaphorical Cognition of Head}

The polysemous senses of "head" are mostly generated by the core sense of "head" through metaphorical cognition 
and the mapping process between the source domain and target domain is based on the people's embodiment experiences. The cultural backgrounds of western countries also plays a key role in the metaphorical cognition of "head". Some metaphorical senses of "head" listed above have distinctive cultural origin, for example, Sense (6) and (13) are closely related to the football culture and the culture of coin in western countries. The cultural factors of these specific metaphorical senses are discussed in the following parts.

\section{A. Coin Culture}

"heads or tails?" is a colloquially expression of the two sides of a coin, which is usually used to ask someone to make an expectation for the result when flipping a coin in the air. In both China and the West, coins, of standard qualities and weights, are normally issued by the official government and function as the medium of exchange or the legal tender. The official government might increase or reduce the issuance of coins according to the demand of the trade. The images on coins are usually well-designed, the side of a coin carrying an image of a monarch or a national emblem is called the obverse ("head"). The other side, with the image of the value and that of a prototypical object of the nation, is called the reverse ("tail").

The obverses ("heads") of European coins are commonly the image of a head portrait of queen or president of the country. For instance, the images on the "heads" of British coins are often the head portrait of Queen Elizabeth II, and the reverses may carry diverse images according to the year of issuance, such as the British National Emblem in 1983, the Scottish thistle in 1984 and the Welsh Leek in 1985, to list just a few. Historically, the head portrait of Washington (the founding president of the United States), Thomas Jefferson (who drafted the Declaration of Independence), Lincoln (the 16th president of the United States) and Franklin Roosevelt (the 32th president of the United States) have appeared in the the obverses ("heads") of American coins. Therefore, the word "head" are endowed with the distinctive sense of "The side of a coin that has a picture of someone's head on it" based on people's experience of coins in western countries.

\section{B. Football Culture}

It is known that modern football is originated from England. Football matches started in England in the beginning of the 12th century, which was held twice a year as a sport of entertainment usually within two cities. Initially, the game was held in the downtown area where the residents lived, the party of the game who kick the ball into the downtown area of the other party would win. If the ball enters the residential house halfway, players would rush in like a swarm of bees and kick the ball disorderly and make a mess of the house. Sometimes pedestrians on the road would suffer a tragic accident when encountering the rolling ball. Later the English government decreed that football matches in downtown areas was banned, which should be held in open space, since then, football field emerged. By the early 19th century, football was quite popular in some European and Latin American countries.

"Heading ball" is a football skill, which refers to the player who hit the ball by certain part of their head, either the right forehead or the side of the forehead with the player remaining on the ground or jumping up. For head is the highest part of the human body and it can touch the ball in the air earlier, the player can fight for much time and take some superiority in the competition by heading the ball. When the word "head" and "ball" appears at the same time, the action of "heading ball" is naturally mapped in people's brain due to people's football experience. Therefore, "head" can be used as a verb with the sense of "To hit a ball with one's head", but it would be hard for people who don't know football and the skill of "hitting ball by head" at all to understand this sense.

\section{Western Beer Culture}

People in most western countries enjoy drinking beer, and beer is well known as "liquid bread". Countries such as America, Britain and Germany possess a long tradition of drinking beer and Germany is one of the biggest beer consumption countries in the world. German beer has a longer shelf life and is more fresh and pure for the production ensures the stability of the components and the sterility of beer at the source and avoiding high temperature and chemical sterilization in the productive process. The wineries along the Rhine River (also called the "Father River") have a long history and profound tradition, and there are various festivals and dances. For instance, the Oktoberfest in Munich, the wealthiest and trendiest city in Germany enjoys a worldwide popularity. According to western people's experience of drinking beer, when the beer is poured into a cup, beer bubbles slowly emerge, and finally rise to the top of the cup. The thick foams in the cup bears a kind of similarity to the spacial feature of "head", therefore, the word "head" get the sense of "The layer of small white bubbles on the beer". Beer was not introduced into China until the early 20th century and people in Chinese rarely categorize the concept of "bear head" into the culture of beer (Feng \& Zhao, 2015).

\section{Geographical Culture}

Cape or headland generally refers to the Long and narrow land protruding from the sea, which is formed by crustal movement combining with the erosion and weathering of sea water. Western countries are mostly maritime countries, The United States borders the Atlantic Ocean to the east and the Pacific Ocean to the west, Canada to the north and Mexico to the south. Britain is an island country, which lies in the North Atlantic Ocean off the north coast of Europe. The English Channel and the North Sea separate Britain from other countries of Europe from the South and north 
respectively. Capes and islands, which are sticking out above the sea, can be seen everywhere in most Western countries, and people commonly associate the state of "Capes and islands raised from the sea" with one's "head" above the shoulders, therefore, the word "head" obtains the the metaphorical sense of "cape or highland on sea" based on people's special and geographical experience.

\section{E. Language Culture}

Languages symbols, either the spoken form or the written one, are tools for people to realize and consolidate their thinking and through which, people realize the exchange of ideas. Language and written symbols of different cultures will inevitably affect people's thinking and understanding of the world. English language is alphabetic writing and characterized by linearity, logicality, flexibility, and constructivity (Wang \& Ren, 2002). Head plays a vital role in the whole human body and when it is mapped into the sentence structure, "head" can be used to stress the central word of a sentence, around which the rest of the parts are organized. In English, the headword (headwords) may appear at the beginning of the sentence, forms the sentence structure of "Head+complement".(for example, he is coming) The headword can also appear in the middle of a sentence, and the element preceding the headword is called the specifier and the element following the headword is named complement, forms the sentence structure of "specifier+Head+complement". (for example, very glad to do) In addition, English is flexible in Word Formation and transformation, word may have different parts of speech. "Head" can be transformed to a verb, an adjective and a noun flexibly. However, "head" is merely used as a noun in many other languages.

\section{F. Factors of Thinking Mode}

Thinking mode is an indirect and general reflection of human brain on the essential attributes and regularity of objective things, and which can be viewed as the product of the social history and culture. A person's thinking mode is gradually shaped and stabilized under the influence of social environment and national culture, and one's actions seems to be influenced by the thinking mode he or she is used to. The unique geographical environment and economic pattern in western regions shaped a linear, open and logical thinking mode of Western people, they are inclined to think and speak candidly and are good at dissecting things and using concrete things to express abstract concepts (Li, 2013). Western people regard "head" as the equivalent of "mind and mental ability" based on the logical analysis of human head: the main function of "head" is thinking and reasoning, with its sub-parts, head controls the operation of the whole body, including one's mental movement and feeling. Therefore, the senses of "thinking" and "mental abilities" are often expressed by "head" through the metaphorical mapping process from the domain of "head" and the domain of "mental activities", so expressions like "Use your head!" "have a good head for business" can make sense (Wang, 2010).

It should be pointed out that, "tou", as the corresponding concept of "head" in Chinese, barely means the top of human organs in most cases without the sense of "thinking and mental abilities". In Chinese, mental activities, such as "imagination", "thinking"and "attention" are often correlated to "xin" (heart), hence there are expressions like "yong xin si kao"(thinking carefully) and "zhuan xin zhi zhi" (be concentrated on doing something). however, the word "tou", combining with "brain", forms the expression of "tou nao" or "nao zi", which can realize the metaphorical references to "intelligence" in Chinese. For instance, the expression of "ta hen you tou nao" means "he who is very smart", the expression of "nao zi hao shi" means that "someone is intelligent and good at thinking".

\section{CONCLUSION}

As cognitive linguists proposed that human thought are largely metaphorical, and metaphors, originated from people's embodiment experience of the physical world are pervasive in people's everyday life. Different nations may have varying Metaphorical Cognition even towards the same thing due to the differences in space-time, region, natural environment and cultural traditions, etc. The word "head" in English has a series of senses, showing the distinctive cognitive mechanisms of western people. After the analysis of different senses of the word "head" from the perspective of conceptual metaphor theory and the further exploration of cultural factors behind the cognition of "head", this paper concluded that the metaphorical cognition of the word "head" is influenced by cultural factors such as Western coin culture, football culture, beer culture, geographical culture, language culture and the modes of thinking etc. This paper is an elementary attempt aims to explore the cultural factors which influence people's Metaphorical Cognition of specific vocabulary in English language, More factors, which contribute to people's Metaphorical Cognition of that vocabulary, like social development and economic growth deserve further analysis, and the Metaphorical Cognition of more vocabularies remain to be explored.

\section{ACKNOWLEDGMENTS}

This paper wouldn't have been completed without the support and help from many people. I d like to express my heartful gratefulness to all of them.

My sincere thanks first go to my supervisor, Mr. Zheng, who provided me with considerable suggestion and guidance since I've decided the topic of the paper. His work style of carefulness and rigorousness always sets a good example in my study.

I also want to express my gratitude to my teacher Miss Liu, it is her concern and encouragement that accompanied 
me during the tough period of preparing this paper, without her support and expectation, I couldn't have finished this work.

I also want to say thanks to My families who are always with me and provide for me a comfortable environment for study.

\section{REFERENCES}

[1] Cheng Wencui. \& Zeng Yanbo. (2003). Reviews on Conceptual Metaphor Study. Journal of Hengyang Normal University (Social Sciences Edition), 4, 118-121.

[2] Deng Haiyan. (2013). Cognitive interpretation of the polysemy of head in terms of metaphor. Academics, 8, 300-307.

[3] Dai Weiping. (2014). Vocabulary \& Metaphor Studies. Beijing: World Publishing Gunagdong Corporation.

[4] Dai Weiping. \& Gao Yanhong. (2007). Metaphor of "Head" in Chinese and English. Guangxi Social Sciences, 3, $137-140$.

[5] Feng Xiangxue. \& Zhao Honglu. (2015). A Comparative study on the Semantic Expansion of Human Body vocabulary in English and Chinese-A Case Study of "Head". Journal of Yan'an Vocational \& Technical Institute, 6, 73-74.

[6] Gao Xiaorong. (2006). A Cognitive Approach to Body Metaphor. Master Degree dissertation, Hebei Normal University.

[7] Li Fenfen. (2013). Comparative Study of Thinking Way between Chinese \& English. Anhui Literature (second half of the month), 4, 56-57.

[8] Lakoff, G. \& Johnson, M. (1980). Metaphors We Live By. Chicago: The University of Chicago Press.

[9] Wang Dan. (2010). A study of head metaphors in English and Chinese. Science \& Technology Information, 8, 131-132.

[10] Wang Xinhua. \& Ren Junli. (2002). A Study of Thinking Way between Chinese \& English and the Cultural Origin. Jiangxi Social Sciences, 9, 43-45.

[11] Xiao Junmin. (2013). A Comparative Study of Human Body vocabulary "Head" in English and Chinese. Anhui Literature, 7 , $131-133$

[12] Zhang Feng. (2004). A Cultural—semantic Analysis of "Head" in Russian and Chinese. Journal of PLA University of Foreign Languages, 3, 96-100.

Man Zheng was born in Zunyi, China in 1995. She received her Bachelor's degree in Commercial English in Zunyi Medical University, China in 2013.

She is currently a master in the school of Foreign languages, GuiZhou Normal University, Guiyang, China. Her research interes ts include cognitive linguistics and English literature. 